\title{
A Zigbee Network Model Used to Large-Scale Networking
}

\author{
$\mathrm{Yu}$ Ren and Kelong $\mathrm{Wu}$ \\ College of Computer, Hangzhou Dianzi University, Hangzhou, China \\ renyu@hdu.edu.cn
}

\begin{abstract}
Zigbee is a kind of wireless network susceptible to interferences. Network relidbility is one of the key elements for the entire network. Zigbee networks have been widely used in many fields such as agriculture, medicine, traffic and environmental protection. The larger the network is, the more complex the environment of network will be, and the move problems will be caused. There are some typical problems, such as the network disconneetion caused by interferences, route errors in communication, the irrationality of networl topology, data congestion appears in the central node of a mesh netmork. These problems are all caused by the instability of network topology eventually, This paper proposses a Zigbee network model used to large-scale networking. The network model maintains a relatively stable network topology, plans the data flow on network links reasonably, andoffers protection for reliable network communication.
\end{abstract}

Keywords: Zigbee, network, communication, model

\section{Introduction}

Since its debut in 2004, there havel been various applications about Zigbee. These applications involve agriculture, traffic, medicine, environmental protection and other fields. Their common characteristic is that the size of network is small. Take the smart home system for example [1], the number of Zigbee nodes is less than 10, and the coverage of network is small, confined to the house. The number of Zigbee nodes in Water environment monitoring system is not large as well [2] In some applications that contain large size of networks, there are always problems. Such as the greenhouse monitoring system in agriculture application [3], the Zigbee nodes in charge of detecting are likely to leave network due to outside interferences, which will lead to the instability of system. By contrastive analysis we find that the larger the size of network, the bigger the likelihood of problems developing.

According to the analysis of its own characteristics, Zigbee is a kind of mesh network susceptible to outside interferences. In the case of small network, when problems arise due to outside interference, these problems are easy to find and have little effect, often be neglected. But in the case of large network, problems caused by outside interferences can evolve into various results, such as data congestion, route errors, devices disconnecting from network. So in practical applications, large-scale network is too unstable to play a due role. One way to solve this problem is to increase the stability of network by reducing data congestion [4]. This method is effective for network with stable topology, but ineffective when devices drop out of network. This paper proposes a new network model. It fundamentally improves the topology of network, strengthens the anti-jamming ability of the network, and makes Zigbee network available for large-scale network fields. 


\section{Network Model}

Zigbee is a kind of self-organizing mesh network with flexibility in network maintenance. In case of disconnecting from the network, nodes can join others network nodes. The capability of self-organizing offers protection for network stability, and it is also the root cause of various problems. For a simple example, as shown in Fig.1 below, routing nodes R1 and R2 join coordinator directly in the initial state. Then the network connection between R2 and coordinator is broken due to outside interferences. After the self-organizing function of Zigbee, node R2 joins coordinator by routing node R1, and new network connection C3 is formed between R1 and R2. On the surface, though there are some changes in network topology, the network still can work. But take the data traffic of network connection into consideration, after the disappearance of $\mathrm{C} 2$ and the appearance of $\mathrm{C} 3$, the data raffic on network connection $\mathrm{C} 1$ doubles, which adds to the instability of $\mathrm{C} 1$ More importantly, Zigbee is just a low speed wireless network. When the data traffic on network connection $\mathcal{1}$ doubles, it may beyond the capacity of $\mathrm{C} 1$. In a word, the larger the size of network is, the greater the probability of problems arising after self-organizing is, and the more serious the consequences will be.

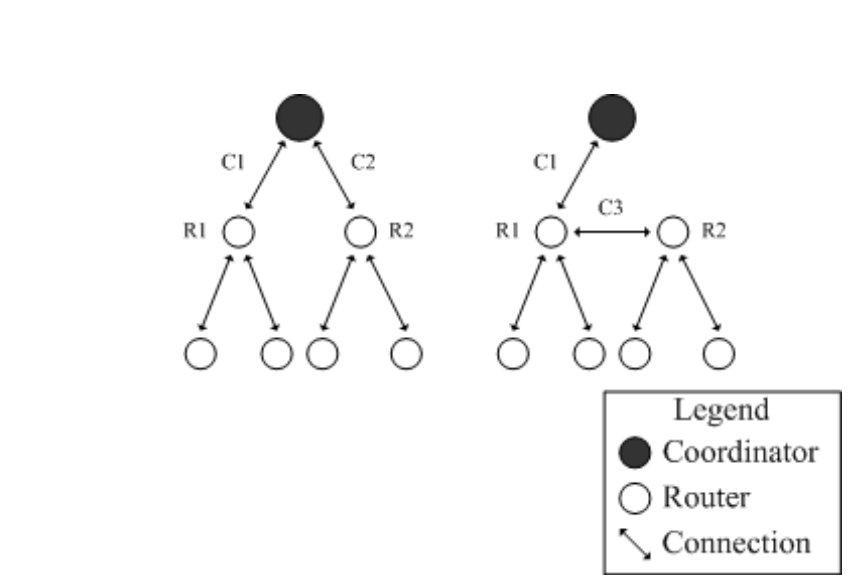

\section{Figure 1. Topological change in Zigbee network}

On the other hand, the self-organizing function of Zigbee has its own limits. When a network node disconnects from original network, if it could not find any node, it will be out of network fofever. In practical application, to offer protection for stable network, it is not enough just to depend on self-organizing function. We should establish a stable backbone network, and all others network nodes can join the backbone network.

The model of backbone network is shown in Figure 2. A backbone network consists of one main coordinator and multiple sets of coordinators and routers. Every set of coordinator and router can communicate by SPI bus directly. The coordinator above and the one below have same channel number and different network number, and the coordinator on the left and the one on the right have incremental channel number and different network number. Every router can receive signals from multiple coordinators except $\mathrm{R} 1$ and $\mathrm{R} 2$. Take router R3 for example, it can join coordinator $\mathrm{C} 1$ or $\mathrm{C} 2$ by selection. The goal of this design is to add stability by increasing the redundancy of backbone network. 


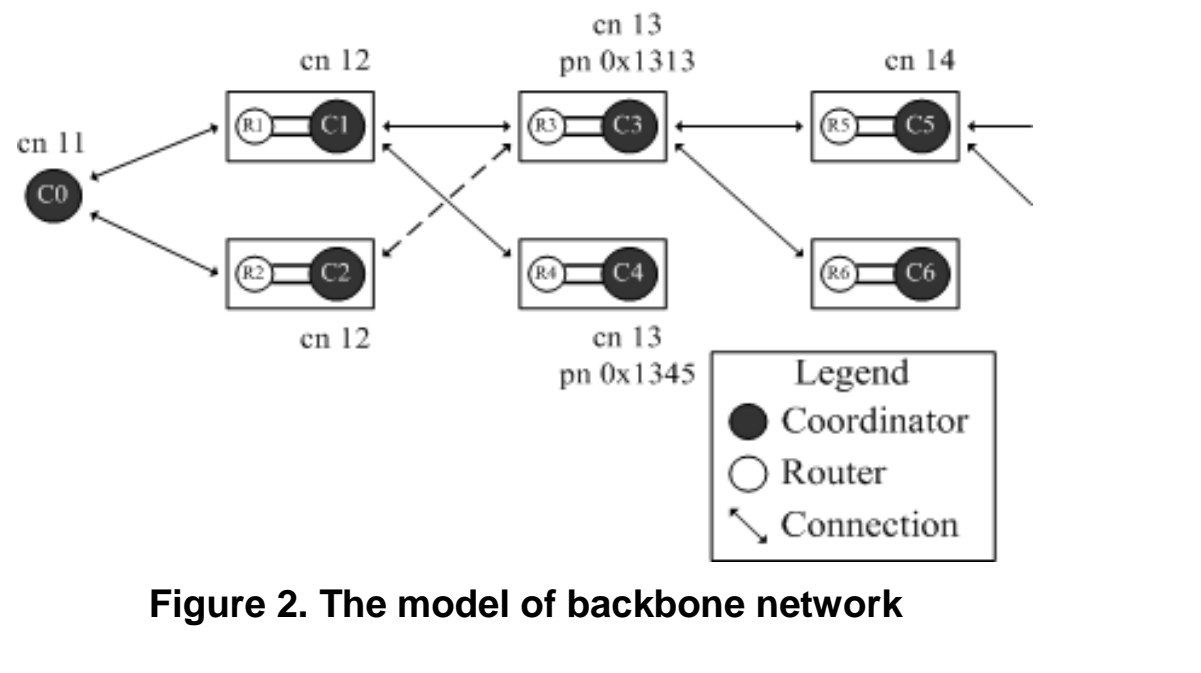

As shown in Figure 3, suppose that coordinator C and router R stop work, as devices malfunction. Router R3, R4 can join coordinator C2 by self-organizing function, and the whole backbone network still can work steadily When the number of corrupted devices is $\mathrm{x}$, the possibility of network failure L can be computed as follows;

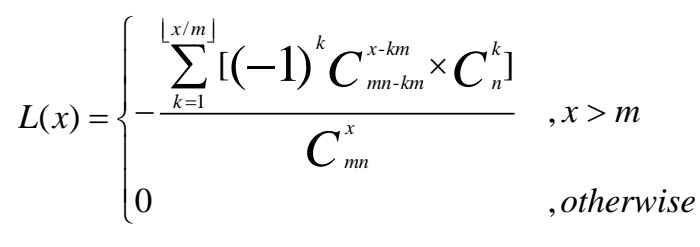

Where $\mathrm{m}$ is the number of rows in the baekbone network, and $\mathrm{n}$ is the number of columns.

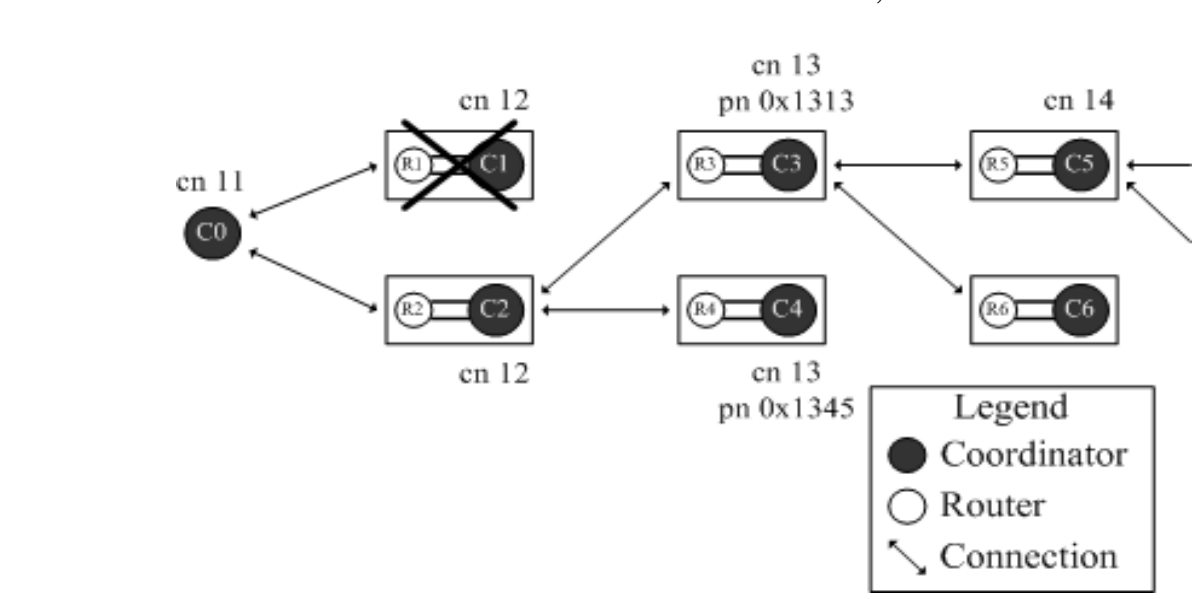

Figure 3. Router R3, R4 rejoin network

After the establishment of backbone network, network nodes can join it. As shown in Figure 4, every coordinator allows nodes to join it, as they are part of backbone network. But when a coordinator is out of backbone network, this coordinator should not allow nodes to join it. Nodes of this coordinator must look for other coordinators. As shown in Figure 5, while coordinator $\mathrm{C} 3$ disconnects from backbone network, and does not allow network nodes to join it, nodes E1, E2, E3 rejoin backbone network by coordinator $\mathrm{C} 4$. In conclusion, the 
characteristic of this network model is that backbone network is responsible for establishing a reliable, stable network, while network nodes join backbone network flexibly.

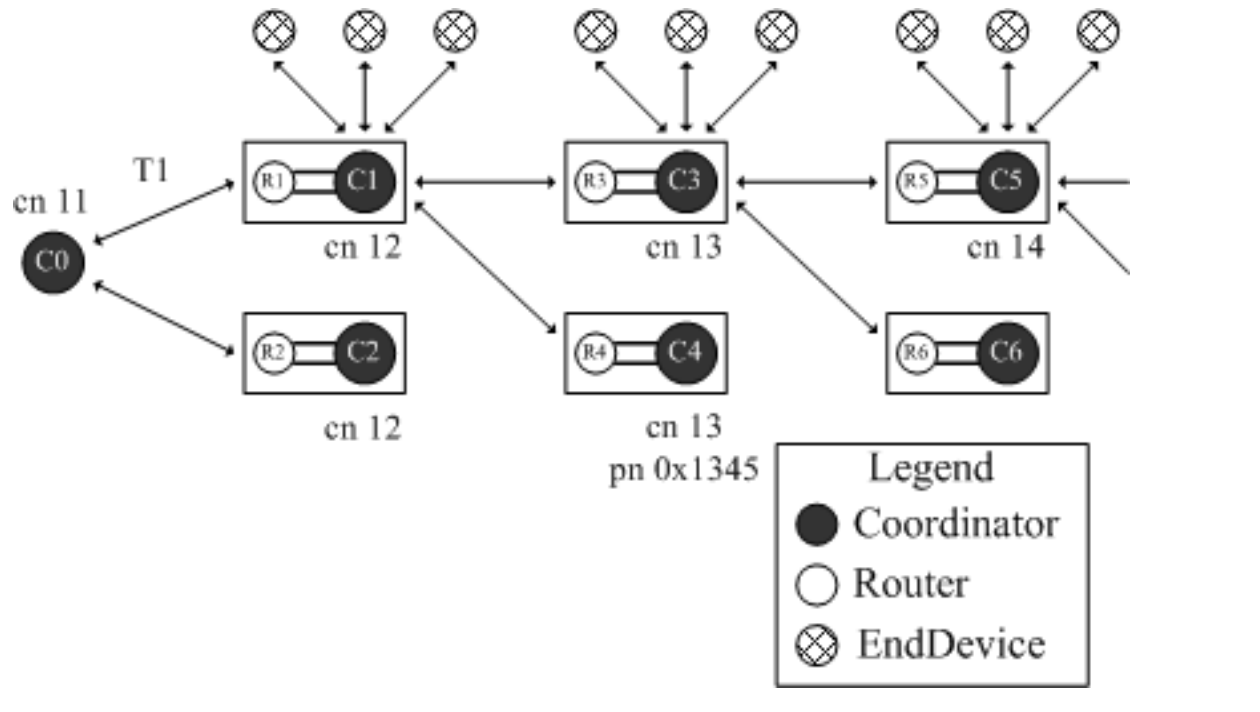

Figure 4. Nodes join backbone network

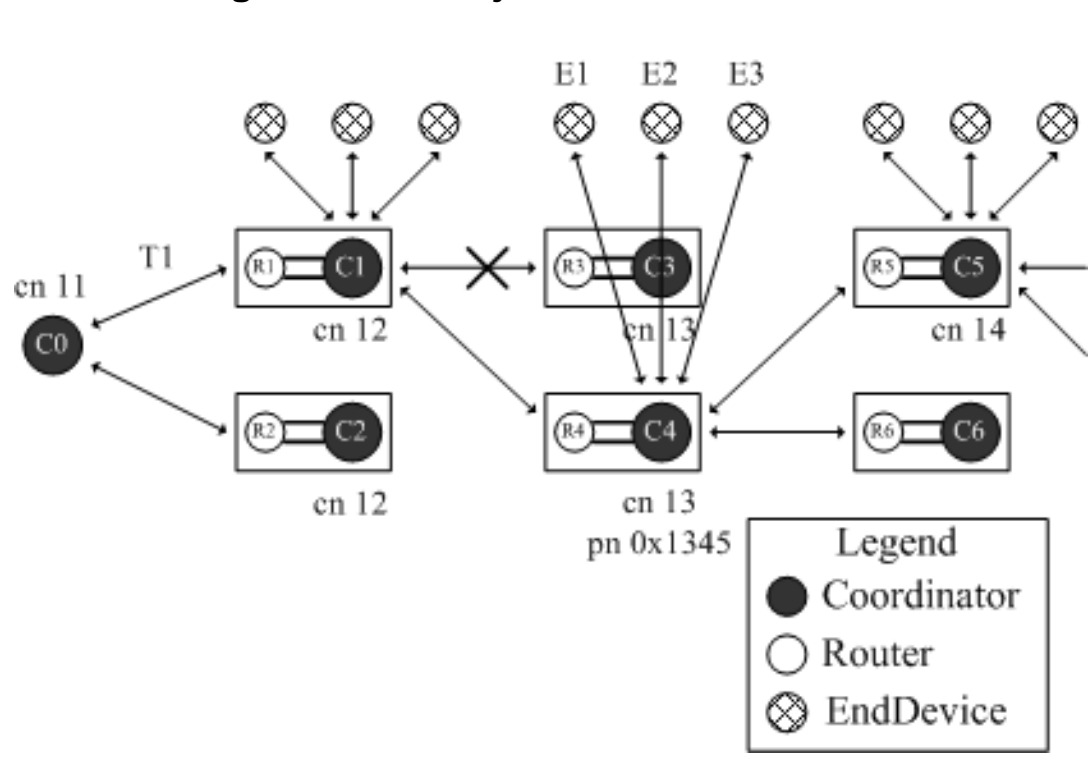

Figure 5. End devices E1,E2 and E3 rejoin network

In this network model, the limit of devices number depends on the maximum communication speed of main coordinator. As shown in Figure 5, the speed of network connection T1 represents the speed of main coordinator. Take one kind of Zigbee network for example, its data throughput is $250 \mathrm{kbps}$. Suppose every node has a communication of $200 \mathrm{bps}$, and in the best case, the network model can hold 1250 nodes. In other word, when the network model contains 1250 nodes, the communication speed on connection T1 is $250 \mathrm{kbps}$. But in practice the data communications of nodes are random and the communication speed of connection T1 fluctuates around $250 \mathrm{kbps}$, that's when the data congestion would happen. So the number of nodes backbone network can hold is far lesson than 1250, about 300 to 500 . 


\section{Test for network model}

To verify the flexibility and the stability of the new network model, an experimental group and a control group are designed. As shown in Figure 6, the experimental group adopts the network model in this article, and it contains a backbone network with 2 rows and 3 columns. Configure end devices E1-E10 to join coordinators C1, C2 only, E11-E20 to join coordinators C3, C4 only, E21-E30 to join coordinators C5, C6 only. As shown in Fig.7, the control group adopts normal Zigbee model, and its end devices E1-E30 are permitted to join all the coordinators. In both groups, end devices E1-E30 send a frame of 10 bytes to the main coordinator every twenty minutes. The main coordinator counts the number of frames it receives every minute. Through this number we can determine the status of network.

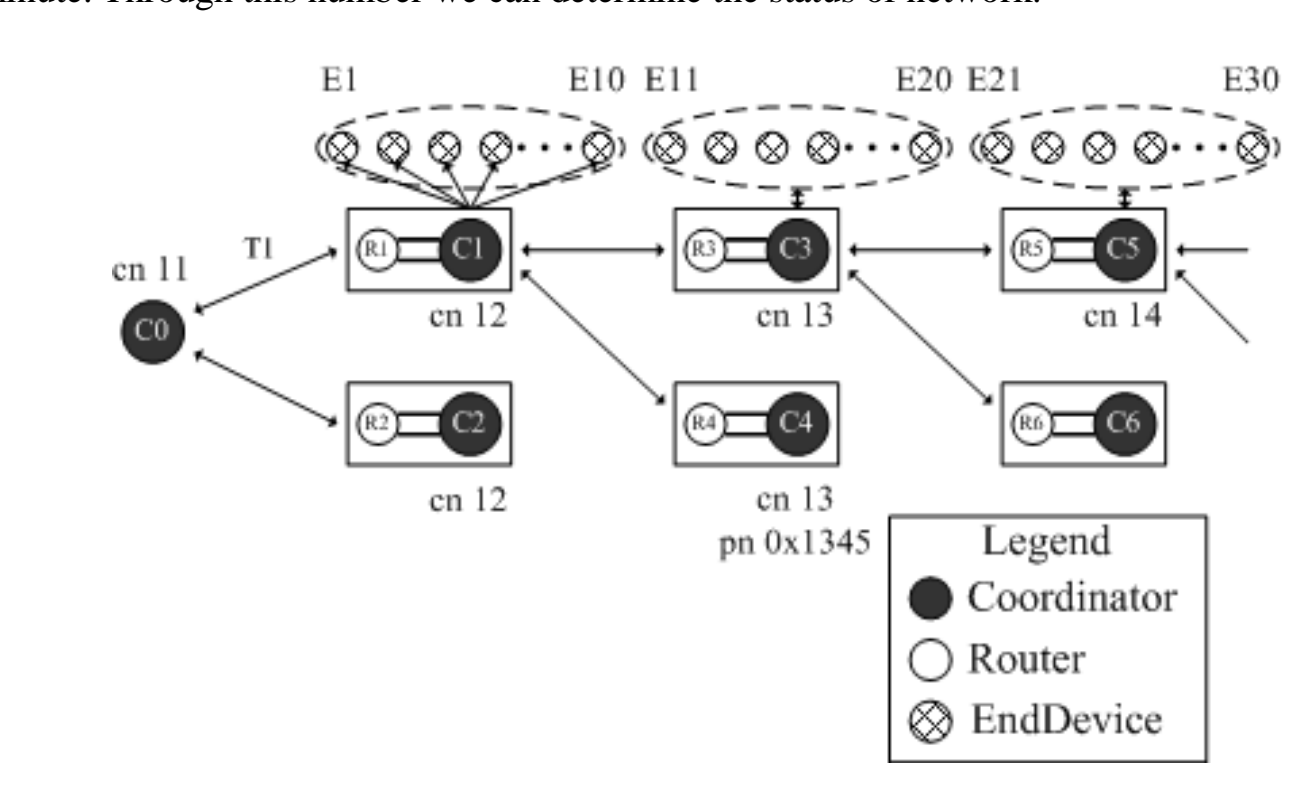

Figure 6. The experimental group with backbone network

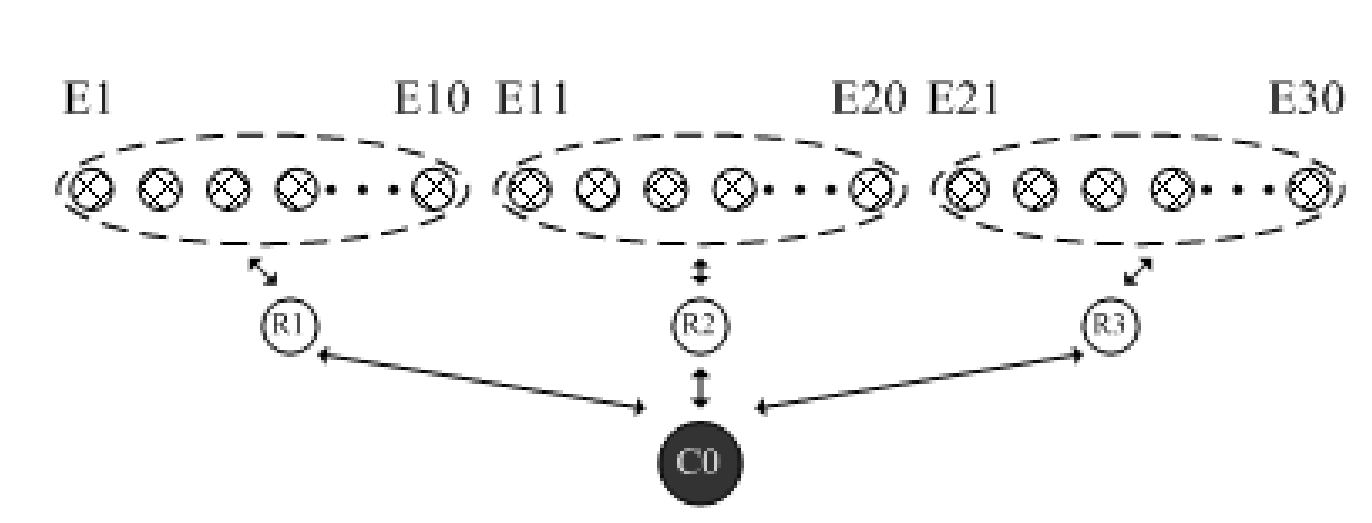

Figure 7. The control group with normal Zigbee model

As shown in Figure 8, the vertical axis represents the number of frames and the horizontal axis represents the time. At the end of the first minute, the number of frames coordinator receives is about 90, which shows that all of 30 end devices join network, and networks of both groups are stable. For the experimental group, at the end of the second minute, we close the 
coordinator $\mathrm{C} 5$. Then at the end of the third minute, the number of frames the main coordinator receives is 60 , and the number of disconnected nodes is 10 . In the fourth, fifth and sixth minutes, this number increases gradually. After the seventh minute, the network tends to be stable. For the control group, at the end of the second minute, we close the router R3. At the end of the third minute, the number of frames the main coordinator receives is 58. In the fourth, fifth minutes, this number increases gradually. After the sixth minute, the network tends to be stable, but compared with experimental group, nodes in network are less and 7 nodes are out of network forever.

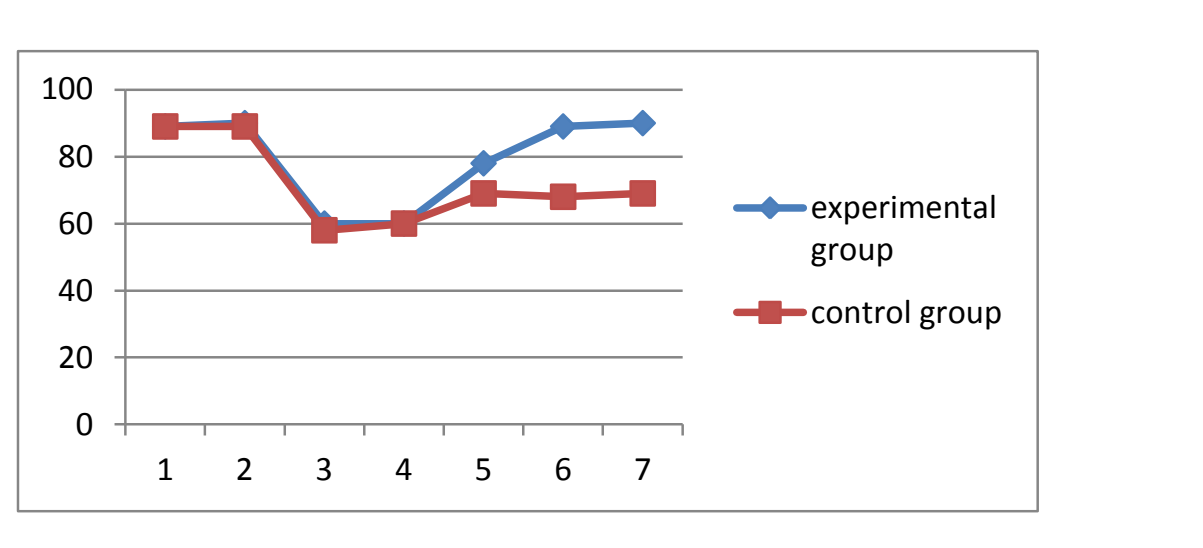

Figure 8. Theresults of edge node failure

As shown in Figure 9, at the end of the second minute, the experimental group closes the coordinator $\mathrm{C} 5$. At the end bf the third ninute, the number of frames the main coordinator receives decreases greatly and most nodes are out of network. After the third minute, the disconnected nodes join network again. For the control group, at the end of the second minute, it closes the router R2. At the end of the third minute, the number of frames coordinator receives decreases, and sonte of nodes are out of network. After the third minute, the nodes join network again, but four of them leave network forever.

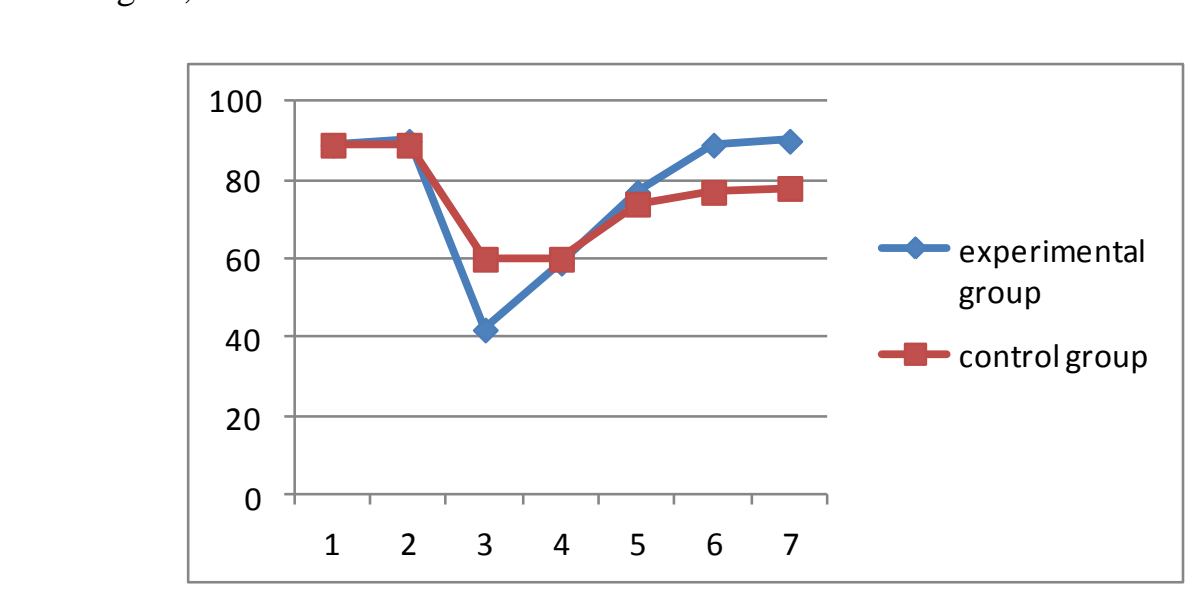

Figure 9. The results of core node failure

As shown in Figure 10, for the experimental group, we close coordinators C3 and C4 at the end of the second minute. At the end of the third minute, the number of frames the main coor- 
dinator receives remains at around 30, and about 20 nodes are out of network forever. For the control group, we close the router R2 at the end of the second minute. At the end of the third minute, the number of frames coordinator receives reduces. After the third minute, this number increases gradually. The network becomes stable finally and 4 nodes are out of network.

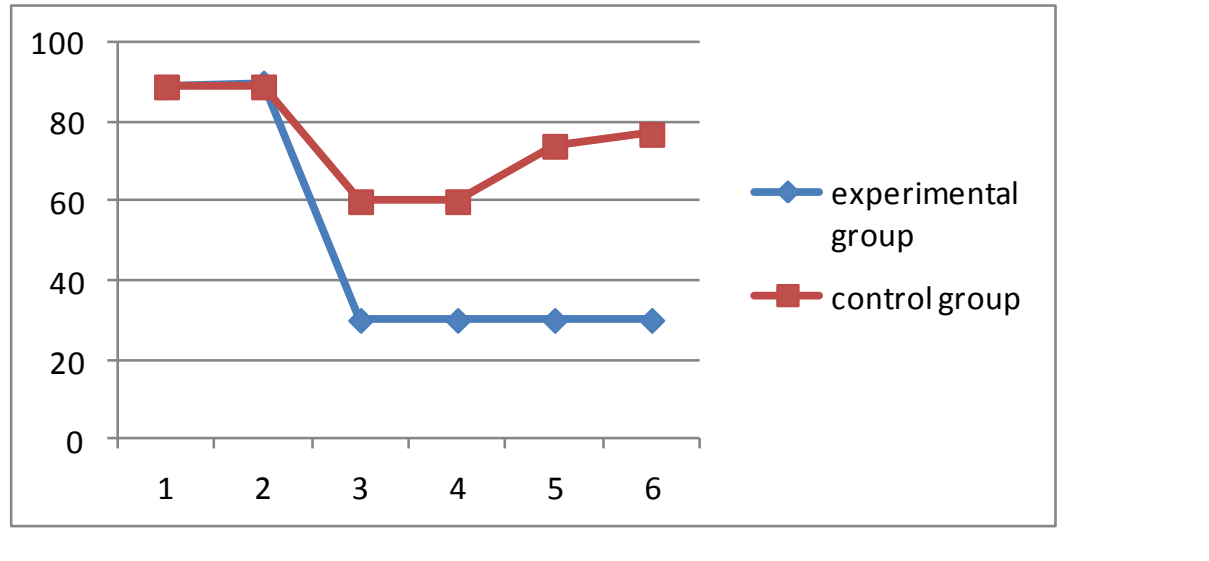

Figure 10. The results of backbone network failure

The results show that, compared with normal Zigbee model, the new network model has greater capability of anti-jamming and self-recoyery But the problem of nodes leaving network is still exist, in the case that all of coordinators in the same column are failed.

\section{Conclusion}

Zigbee is a network with complicated and changing topology, which is inconvenient for practical application. This paper proposes a new kind of network model. This model simplifies the topology of network (by)establishing backbone network and increases the flexibility of nodes by self-organizing function. Through practical test, we can insure that the stability and the flexibility of this model can provide support for practical application.

\section{References}

[1] Z-Dexing, J. Wei, L. Yongli, H. Jiuqiang and L. Shengbin, "An improved routing algorithm of Zigbee wireless sensor network for smart home system", Proceedings of the 5th International Conference on Automation, Robotics and Applications, (2011), pp. 346-350.

[2] L. Haifeng and H. Xufeng, "Water environment monitoring system based on Zigbee technology", Proceedings of the 2013 3rd International Conference on Intelligent System Design and Engineering Applications, ISDEA, (2013), pp. 1607-1609.

[3] M. Ma and G. Feng, "Based on Zigbee Technology Greenhouse Monitoring System", Proceedings of the 2012 International Conference of Modern Computer Science and Applications, Advances in Intelligent Systems and Computing, vol. 191, (2013), pp. 737-740.

[4] D. S. Yun, S. H. Cho, D. W. Seo and M. S. Kang, "An efficient and reliable data transmission control method for relaxing congestion problem in ZigBee network", Proceedings of the 2nd International Conference on Ubiquitous Information Management and Communication, ICUIMC-2008, (2008), pp. 533-537. 


\section{Authors}

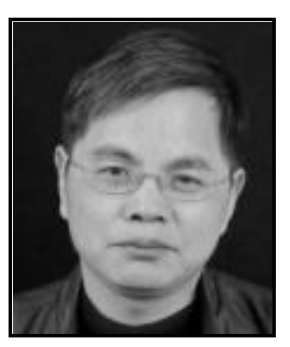

\section{Yu Ren}

He received the Bachelor Degree of Mechanic Engineering from Zhejiang University, China, in July 1985, and the Master Degree of Computer Graphic from Zhejiang University, in Dec.1989. His research interests include embedded application system, real time control system and the thing of internet.

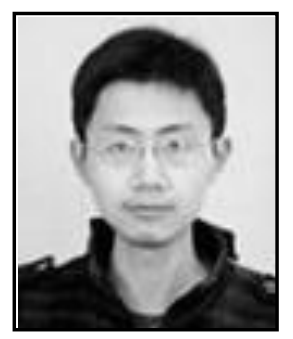

\section{Kelong Wu}

He received the Bachelor Degree of Computer Science and Technology from Hangzhou Dianzi University, China, in June 2007, and started postgraduate study at Hangzhou Dianzi University. He has been working on embedded software programmed and designed for three years.

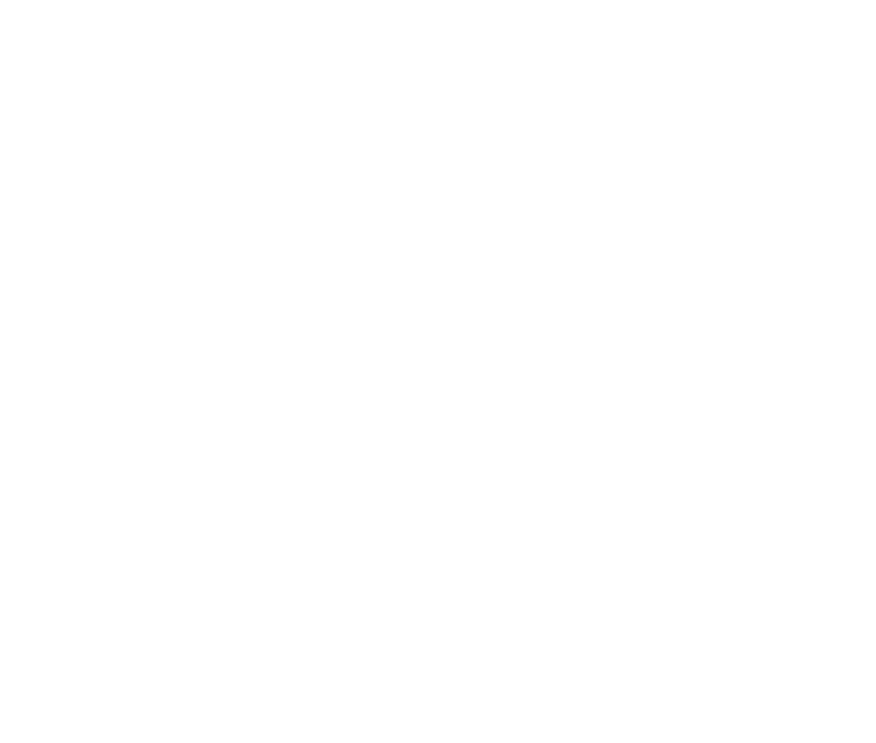

